\title{
Shadow of a charged rotating non-commutative black hole
}

\author{
M. Sharif ${ }^{1,2, a}$, Sehrish Iftikhar ${ }^{1, b}$ \\ ${ }^{1}$ Department of Mathematics, University of the Punjab, Quaid-e-Azam Campus, Lahore 54590, Pakistan \\ 2 Pakistan Academy of Sciences, 3 Constitution Avenue, G-5/2, Islamabad, Pakistan
}

Received: 9 August 2016 / Accepted: 19 October 2016 / Published online: 18 November 2016

(c) The Author(s) 2016. This article is published with open access at Springerlink.com

\begin{abstract}
This paper investigates the shadow of a charged rotating non-commutative black hole. For this purpose, we first formulate the null geodesics and study the effects of a non-commutative charge on the photon orbit. We then explore the effect of spin, angle of inclination as well as noncommutative charge on the silhouette of the shadow. It is found that shape of the shadow deviates from the circle with the decrease in the non-commutative charge. We also discuss observable quantities to study the deformation and distortion in the shadow cast by the black hole which decreases for small values of a non-commutative charge. Finally, we study the shadows in the presence of plasma. We conclude that the non-commutativity has a great impact on the black hole shadow.
\end{abstract}

\section{Introduction}

Black holes (BHs) are the end products of a complete gravitational collapse of the massive star surrounded by a boundary from which nothing can escape even light. Despite their dark nature, BHs exist in the environment of the brightest objects of the universe. Black hole does not shine but the accreting gas that spirals around, emits radiations and then disappears when reaches the event horizon. Generally, a BH casts a shadow in an emitting medium which arises due to the bending of light that originates near the horizon and travels through the accretion flow. The shape of a shadow depends on the photon capture sphere resulting from strong bending of light perceived by a distant observer. The shadow can actually depict the compact object (BH or naked singularity) as its appropriate observations can provide information as regards

S. Iftikhar: On leave from Department of Mathematics, Lahore College for Women University, Lahore-54000, Pakistan.

\footnotetext{
a e-mail: msharif.math@pu.edu.pk

b e-mail: sehrish3iftikhar@gmail.com
}

the parameters as well as spacetime geometry around the compact object.

Black holes are usually of two types: stellar mass BHs that are observed when they accrete matter from X-ray binaries and the supermassive BHs in the center of galaxies. The direct observation of $\mathrm{BH}$ is not possible, therefore, the event horizon is the defining characteristic which casts a relatively large shadow with respect to a distant observer. The interest in this topic was raised with the possibility of viewing the shadow of Sgr A*, a central supermassive BH candidate of the Milky way. It is shown that the shadow of Sgr A* is observable with very long baseline interferometry at submillimeter wavelengths with the assumption of thin accretion flow in the related region [1]. Further developments in this framework can be viewed in the literature [2-5].

The concept of a BH shadow initiated with Bardeen [6] as well as Cunningham and Bardeen [7] who studied shadow of the Kerr BH. This technique can also be found in [8], where coordinates of the observer's sky have been calculated. Different aspects related to shadows for the Kerr-Newman BH were discussed in [9-11]. Hioki and Maeda [12] discussed that $\mathrm{BH}$ can be distinguished from naked singularity by examining the shadows of Kerr BH as well as naked singularity. They provided a technique to measure the spin and angle of inclination by defining two observables. The properties of different BHs shadows have been extensively studied in the literature [13-18]. The shadow of binary BHs is another interesting subject. Bohn et al. [19] showed that the shadow of binary BHs is different from the superposition of two singleton BH shadows. Shipley and Dolan [20] explored the shadows of two extremely charged binary BHs and stated that they are interesting examples of chaotic scattering in nature. Another fascinating feature of the $\mathrm{BH}$ shadows is that it can serve to test the no hair theorem [21-23].

The occurrence of curvature singularities in gravitational collapse has led to important developments in general relativity (GR). To resolve the problem of singularities, quantum gravity proved to be very successful. Since GR breaks down 
at short distances, the non-commutative (NC) theory provides a useful approach to discuss the spacetime dynamics at Planck scale. Gross and Mende [24,25] explored the behavior of string interactions at short distances. Maggiore [26] found a generalized uncertainty principle which is consistent with the results obtained in string theory. He suggested that the minimum length (of order Planck length) emerges naturally from any theory of quantum gravity. The structure of $\mathrm{NC}$ geometry is based on a universal minimal length scale which is equivalent to Planck's length. In this framework, the spacetime quantization requires that its coordinates become Hermitian operators which do not commute, i.e., [27]

$\left[x^{A}, x^{B}\right]=\iota \Theta^{A B}$,

where $\Theta^{A B}$ is a real-valued antisymmetric matrix with $\Theta^{A B}=\Theta \operatorname{diag}\left(\epsilon_{i j}, \epsilon_{i j}, \ldots\right), \Theta$ is a constant of dimension (length) $)^{2}$. In the limit $\Theta \rightarrow 0$, the ordinary spacetime is recovered. Physically, $\Theta^{A B}$ represents a small patch in $A B$ plane of the observable area as the Planck constant $(\hbar)$ illustrates the smallest fundamental cell of the observable phase space in quantum mechanics.

The quantum gravity BHs incorporates the effects of quantum gravity in the vicinity of origin, where the classical curvature singularity appears. Many singularity free $\mathrm{BHs}$ have been obtained in the framework of quantum gravity such as BHs inspired by NC geometry. In the early 1990s, Doplicher et al. $[28,29]$ provided a detailed study of the spacetime non-commutativity which shows that GR together with the uncertainty principle of quantum mechanics leads to a class of models of $\mathrm{NC}$ spacetime. There are some comprehensive reviews of NC field theory and the spacetime with NC coordinates $[30,31]$. The $\mathrm{NC}$ form of different $\mathrm{BHs}$ such as charged [32], higher dimensional [33,34], and rotating [35] cases have also been obtained. The effects of non-commutativity on the properties of BHs have extensively been studied. Nicolini et al. [36] investigated a radiating NC Schwarzschild BH and found that non-commutativity can cure singularity problems. Nozari and Mehdipour [37] explored quantum tunneling from an NC Schwarzschild BH and showed that the information would be preserved by a stable $\mathrm{BH}$ remnant due to $\mathrm{NC}$ effects. The lensing properties of $\mathrm{NC} \mathrm{BHs}$ have also been explored. Ding et al. [38] studied the influence of the NC parameter on strong field lensing of the NC Schwarzschild $\mathrm{BH}$ and found that the $\mathrm{NC}$ parameter has a similar behavior to that of the charge of a Reissner-Nordström BH. Wen-Wei [39] found that the NC parameter affects the shape of the $\mathrm{BH}$ shadow and increases its deformation as compared to the Kerr BH. Many other important aspects of spacetime noncommutativity have been discussed in the literature [40-44].

Generally, BHs are characterized by non-zero angular momentum which breaks the spherical symmetry resulting in rotational symmetry around axis. The gravitational field around a BH is highly affected by its rotation, therefore, it is natural to expect some important effects on the shadow cast by the BH. It is observed that the shadow of a non-rotating $\mathrm{BH}$ has a circular shape while the shadow of a rotating $\mathrm{BH}$ is deformed due to its spin $[8,12,45]$. Another motivation comes from Sgr A* - the most remarkable candidate for a $\mathrm{BH}$ which likely has a spin whose axis is perpendicular to the galactic plane. The predicted size of the shadow of Sgr A* is $\sim 30 \mu$ arcseconds [1]. According to the no hair theorem, any stationary, asymptotically flat $\mathrm{BH}$ vacuum solution of the field equations can be characterized by three variables: mass, charge, and angular momentum per unit mass $(M, Q, a)$. This leads to the formation of a Kerr-Newman (KN) BH as an outcome of non-spherical gravitational collapse. It is suggested that an isolated rotating BH cannot have an electromagnetic field except if it is endowed with a net electric charge [46-48]. It is possible that a Kerr-Newman BH could be the end point of magnetized, massive rotating stars as a result of catastrophic gravitational collapse [49]. It is also proposed that the charged rapidly rotating galactic $\mathrm{BHs}$ (Kerr Newman BHs) emit gamma rays (in a bipolar out flow) [50].

The effect of plasma on the properties of $\mathrm{BH}$ has extensively been studied. Muhleman and Johnston [51] discussed the deflection of light rays by gravity as well plasma by considering radio waves traveling near the Sun. He used a product of the plasma and gravitational refractive indices in the weak field approximation. Breuer and Ehlers [52,53] explored the Hamiltonian for light rays in a magnetized pressureless plasma. A similar derivation was found by Perlick [54] for the pressureless, non-magnetized plasma. Perlick et al. [55] discussed the effect of plasma on the shadow of Ellis wormholes as well as spherically symmetric $\mathrm{BH}$ and found that the decrease in the shadow by the presence of plasma. Rogers [56] explored gravitational lensing and circular orbits of the Schwarzschild $\mathrm{BH}$ for plasma density distributions $N=1 / r^{h}$ with $h \geq 0$. There is a large body of literature available [57-62] on the effect of plasma on the shadows cast by the BHs.

In this paper, we study the shadow of charged rotating $\mathrm{NC}$ $\mathrm{BH}$ in the equatorial as well as non-equatorial plane. The paper is organized as follows. In the next section, we discuss the null geodesics for the $\mathrm{NC} \mathrm{BH}$. Section 3 is devoted to the study of the apparent shape of the NC geometry inspired shadows and the behavior of observables. In Sect. 4, we explore the influence of plasma on the shape of shadows. Final results and conclusions are in the last section.

\section{Geodesics in a non-commutative spacetime}

Non-commutativity is an intrinsic property of the spacetime which implies the existence of a natural ultra-violet cutoff or equivalently a minimal (measurable) length in quantum 
field theory. The NC spaces have a long history. The first model of NC spacetime (admitting a minimal length scale and invariant under the Lorentz transformation) was proposed by Snyder [63,64] which was an attempt to introduce a short distance cutoff in quantum field theory. The interest in NC theory increased with the seminal paper of Seiberg and Witten [65] in which they extended NC theory to the string theory with a constant $B$-field. Some important examples of non-commutativity include Lei algebra type deformation (the most studied example is $\kappa$-Minkowski spacetime $[66,67]$ ) and canonical type deformation (a simple and widely discussed example of $\mathrm{NC}$ spacetime) $[28,65,68,69]$. In NC geometry, the pointlike smeared gravitating source ( $\rho_{\Theta}(r)=\frac{1}{(4 \Theta)^{\mathrm{d} / 2}} e^{-x^{2} / 4 \Theta}, d$ is the dimension of spacetime) is assumed to remove divergencies in GR. Modesto and Nicolini [70] obtained a regular charged rotating $\mathrm{BH}$ solution in the background of $\mathrm{NC}$ geometry (based on a canonical deformation) by employing the Newman-Janis mechanism. They found that the resulting BH solution is unique and exhibits a local regular behavior at the origin as well as standard rotating geometries at large distances. The Ricci scalar as well as the Kretschmann invariant remain finite at the origin. The charged NC BH in Boyer-Lindquist coordinates is given as

$$
\begin{aligned}
\mathrm{d} s^{2}= & \left(\frac{\Delta-a^{2} \sin ^{2} \theta}{\Sigma}\right) \mathrm{d} t^{2}-\frac{\Sigma}{\Delta} \mathrm{d} r^{2}-\Sigma \mathrm{d} \theta^{2} \\
& +2 a \sin ^{2} \theta\left[1-\frac{\Delta-a^{2} \sin ^{2} \theta}{\Sigma}\right] \mathrm{d} t \mathrm{~d} \phi \\
& -\sin ^{2} \theta\left[\Sigma+a^{2} \sin ^{2} \theta\left(2-\frac{\Delta-a^{2} \sin ^{2} \theta}{\Sigma}\right)\right] \mathrm{d} \phi^{2},
\end{aligned}
$$

where

$\Delta=r^{2}+a^{2}-2 m(r) r+q^{2}(r), \quad \Sigma=r^{2}+a^{2} \cos \theta^{2}$,

$m(r)=\frac{M \gamma\left(\frac{3}{2} ; \frac{r^{2}}{4 \Theta}\right)}{\Gamma\left(\frac{3}{2}\right)}, \quad q^{2}(r)=\frac{Q^{2}}{\pi}$

$\times\left[\gamma^{2}\left(\frac{1}{2} ; \frac{r^{2}}{4 \Theta}\right)-\frac{r}{\sqrt{2 \Theta}} \gamma\left(\frac{1}{2} ; \frac{r^{2}}{2 \Theta}\right)+r \sqrt{\frac{2}{\Theta}} \gamma\left(\frac{3}{2} ; \frac{r^{2}}{4 \Theta}\right)\right]$.

The event horizon of (1) is the largest root of $\Delta=0$ given by

$r^{2}+a^{2}-2 m(r) r+q^{2}(r)=0$.

A detailed discussion as regards the nature of horizons and thermodynamical properties are available in [70]. Here we would like to discuss the horizons briefly. The function $\Delta=0$ produces horizons for $M \geq M_{\text {extr }}$, i.e., for extremal and non-extremal cases, (1) represents a BH but for $M<M_{\text {extr }}$, there is no horizon and the metric (1) describes a regular charged spinning object such as charged spinning mini gravastar [70]. In this paper, we do not consider the horizonless case. For this purpose, we find maximum values for the spin and charge parameter corresponding to different values of the non-commutative parameter by solving $\Delta(r)=0$ and $\frac{\mathrm{d} \Delta(r)}{\mathrm{d} r}=0$ numerically (Fig. 1).

The particle motion can be described by the Lagrangian

$\mathcal{L}=\frac{1}{2} g_{v \sigma} \dot{x}^{\nu} \dot{x}^{\sigma}$,

where $\dot{x}^{\nu}=u^{\nu}=\mathrm{d} x^{\nu} / \mathrm{d} \lambda, u^{\nu}$ is the particle's 4-velocity and $\lambda$ is the affine parameter. The energy $E$ and angular momentum $L$ are given by

$$
\begin{aligned}
& E=p_{t}=\frac{\partial \mathcal{L}}{\partial \dot{t}}=g_{\phi t} \dot{\phi}+g_{t t} \dot{t}, \\
& L=-p_{\phi}=-\frac{\partial \mathcal{L}}{\partial \dot{\phi}}=-g_{\phi \phi} \dot{\phi}-g_{\phi t} \dot{t} .
\end{aligned}
$$

The Hamilton-Jacobi equation yields

$$
\frac{\partial S}{\partial \lambda}=\frac{1}{2} g^{\nu \sigma} \frac{\partial S}{\partial x^{\nu}} \frac{\partial S}{\partial x^{\sigma}} .
$$

We find that the Lagrangian is independent of $t$ and $\phi$, therefore $p_{t}$ and $p_{\phi}$ are conserved and hence describe the stationary and axisymmetric characteristics of the charged rotating NC BH. Equation (4) takes the form

$S=\frac{1}{2} m_{0}^{2} \lambda-E t+L \phi+S_{r}(r)+S_{\theta}(\theta)$.

For the metric (1), it leads to

$\Sigma \frac{\partial t}{\partial \lambda}=a\left(L-a E \sin ^{2} \theta\right)+\frac{r^{2}+a^{2}}{\Delta}\left[E\left(r^{2}+a^{2}\right)-a L\right]$,

$\Sigma \frac{\partial \phi}{\partial \lambda}=\left(L \csc ^{2} \theta-a E\right)+\frac{a}{\Delta}\left[E\left(r^{2}+a^{2}\right)-a L\right]$,

$\Sigma \frac{\partial r}{\partial \lambda}= \pm \sqrt{R}$

$\Sigma \frac{\partial \theta}{\partial \lambda}= \pm \sqrt{\Theta}$,

where

$R=\left[E\left(r^{2}+a^{2}\right)-a L\right]^{2}-\Delta\left[K+(L-a E)^{2}\right]$,

$\Theta=K+\cos ^{2} \theta\left(a^{2} E^{2}-L^{2} \csc ^{2} \theta\right)$,

and $K$ is the separation constant introduced by Carter [76]. Equation (8) can be represented as

$$
\left(\Sigma \frac{\partial r}{\partial \lambda}\right)^{2}+V_{\mathrm{eff}}=0
$$

where

$V_{\text {eff }}=\left[E\left(r^{2}+a^{2}\right)-a L\right]^{2}-\Delta\left[K+(L-a E)^{2}\right]$. 

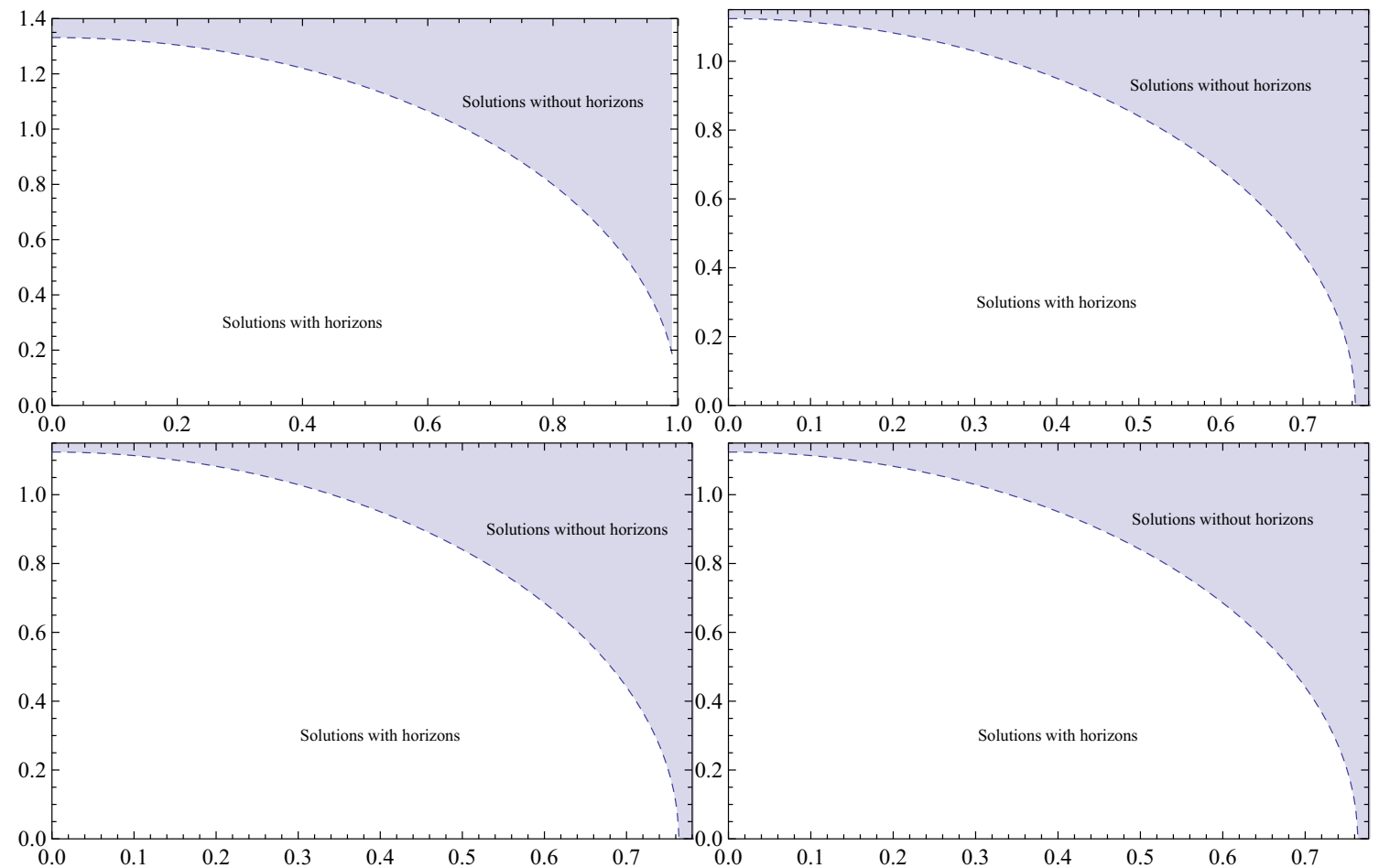

Fig. 1 The parameter space indicates the charged rotating $\mathrm{NC} \mathrm{BH}$. Here, the vertical axis shows $Q / M$ and the horizontal axis represents $a / M$. The blue line is the boundary that separates the region of $\mathrm{BH}$ from
non-BH. The upper panel plots correspond to $\Theta=0.1$ (left), $\Theta=0.2$ (right) while lower panel to $\Theta=0.3$ (left), $\Theta=0.4$ (right)

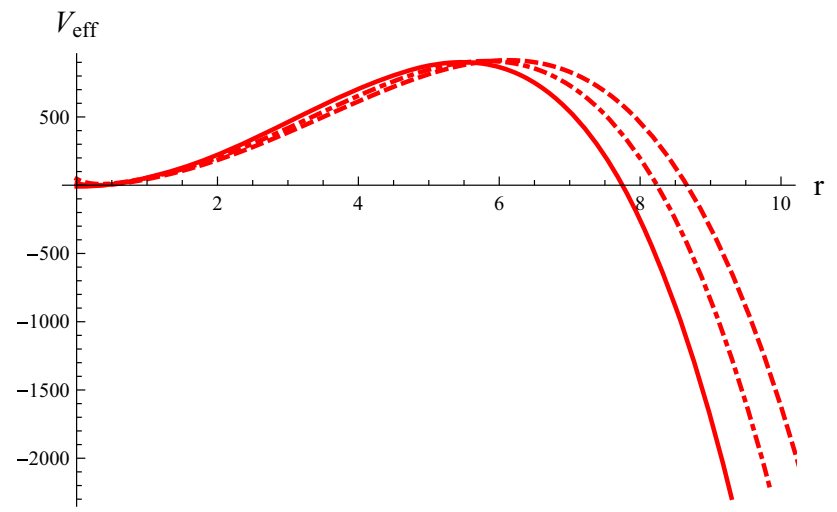

Fig. 2 Plots of the effective potential versus $r$ corresponding to $Q=$ 0.2 (solid), $Q=0.3$ (dashed) and $Q=0.5$ (dot-dashed) with $\Theta=0.3$ and $a=0.2$

The above equation can describe radial motion of the particles. Figure 2 shows that orbits of the photons are initially unstable for small values of $r$, it approaches to maximum values (which shows the stable orbits) and finally becomes unstable with increasing $r$. We also note that stable points for the photons in the charged NC geometry corresponding to large values of charge are greater than that of small values of charge.

The conditions for unstable circular orbit $R(r)=0$ and $\frac{\mathrm{d} R(r)}{\mathrm{d} r}=0[8]$ lead to

$$
\begin{aligned}
& r^{4}+\left(a^{2}-\xi^{2}-\eta\right) r^{2}+\left(2 m(r) r-q^{2}(r)\right) \\
& \quad \times\left(\eta+(\xi-a)^{2}\right)-a^{2} \eta=0, \\
& 4 r^{3}+2\left(a^{2}-\xi^{2}-\eta\right) r+\left(2 m^{\prime}(r) r+2 m(r)-2 q(r) q^{\prime}(r)\right) \\
& \quad \times\left(\eta+(\xi-a)^{2}\right)=0,
\end{aligned}
$$

where $\xi=L / E, \eta=K / E^{2}$ are the impact parameters and

$$
\begin{aligned}
m^{\prime}(r)= & -\frac{e^{-\frac{r^{2}}{4 \Theta}} M r \sqrt{\frac{r^{2}}{\Theta}}}{2 \Theta \sqrt{\pi}}, \\
q^{\prime}(r)= & \frac{Q^{2}}{\pi}\left[\frac{e^{\frac{r^{2}}{2 \Theta r^{2}}}}{\Theta^{3 / 2} \sqrt{\frac{r^{2}}{\Theta}}}-\frac{\sqrt{2} r^{2} e^{\frac{r^{2}}{2 \Theta r^{2}}}}{\Theta^{3 / 2} \sqrt{\frac{r^{2}}{\Theta}}}+\sqrt{2} \sqrt{\frac{1}{\Theta}} \gamma\left(\frac{1}{2}, \frac{r^{2}}{4 \Theta}\right)\right. \\
& \left.-\frac{2 r e^{\frac{r^{2}}{2 \Theta r^{2}}} \gamma\left(\frac{1}{2}, \frac{r^{2}}{4 \Theta}\right)}{\Theta \sqrt{\frac{r^{2}}{\Theta}}}-\frac{\gamma\left(\frac{1}{2}, \frac{r^{2}}{2 \Theta}\right)}{\sqrt{2 \Theta}}\right] .
\end{aligned}
$$

Solving Eqs. (13) and (14), we have

$$
\begin{aligned}
\xi= & \frac{1}{a\left[m(r)+r\left(m^{\prime}(r)-1\right)-q(r) q^{\prime}(r)\right]}\left[m(r)\left(a^{2}-3 r^{2}\right)\right. \\
& +r\left(r^{2}+a^{2}\right) \\
& \left.\times\left(m^{\prime}(r)+1\right)+2 q^{2}(r)-q(r) q^{\prime}(r)\left(r^{2}+a^{2}\right)\right],
\end{aligned}
$$




$$
\begin{aligned}
\eta= & \frac{r^{2}}{a^{2}\left[m(r)+r\left(m^{\prime}(r)-1\right)\right]^{2}}\left[m(r)\left(4 a^{2}-9 m(r) r+6 r^{2}\right)\right. \\
& \times r-2 m^{\prime}(r) \\
& \times\left(2 a^{2}+r^{2}-3 m(r) r\right) r^{2}-r^{4}\left(m^{\prime 2}(r)+1\right)-4 q^{2}(r) \\
& \times\left(a^{2}+q^{2}(r)-3 m(r) r+m^{\prime}(r) r^{2}+r^{2}\right)-q^{\prime}(r) \\
& \times\left(4 a^{2}+4 q^{3}(r)\right. \\
& \left.\left.-6 m(r) q^{\prime}(r) r-2 m^{\prime}(r) q(r) r^{2}-q(r) r+2 q(r) r^{2}\right)\right] .
\end{aligned}
$$

The above equations are important as they are related to the celestial coordinates of the image seen by an observer at infinity and determine the contour of the $\mathrm{BH}$ shadow (this will be discussed in the next section).

\section{Some features of black holes}

Here, we study some features of BHs related to its shadows.

\subsection{Shadows}

Photons that emit from a background source of a BH suffer deflection due strong gravitational field. The photons having small impact parameter fall into the $\mathrm{BH}$ and form a dark region in sky. An observer viewing that part of the sky would observe a black spot having larger radius than the event horizon around the BH. This dark region is called the shadow of the $\mathrm{BH}$. The shape of the $\mathrm{BH}$ shadow for a far away observer can be determined by the following celestial coordinates [45]:

$$
\begin{aligned}
& \alpha=\lim _{r \rightarrow \infty}\left(-\left.r^{2} \sin \theta \frac{d \phi}{d r}\right|_{\theta \rightarrow \theta_{0}}\right), \\
& \beta=\lim _{r \rightarrow \infty}\left(\left.r^{2} \frac{d \theta}{d r}\right|_{\theta \rightarrow \theta_{0}}\right),
\end{aligned}
$$

where $\theta_{0}$ is the angle of inclination between the rotation axis of the BH and the observer's line of sight. The celestial coordinates $\alpha$ and $\beta$ represent the apparent perpendicular distances of the image around the $\mathrm{BH}$, seen from the axis of symmetry and from its projection on equatorial plane, respectively. These coordinates provide the apparent position of the image in the plane which passes through the center of $\mathrm{BH}$ and is orthogonal to the line joining the $\mathrm{BH}$ and observer. Using Eqs. (7)-(9) in (17)-(18), we have

$\alpha=-\xi \csc \theta_{0}$,

$\beta=\sqrt{\eta+a^{2} \cos ^{2} \theta_{0}-\xi^{2} \cot ^{2} \theta_{0}}$.

Here $\xi$ and $\eta$ are given by Eqs. (15)-(16). The shadow of a charged rotating NC BH is shown in Fig. 3. The silhouette of the shadow is influenced by NC mass, charge, spin, and the angle of inclination. The shape of the shadow changes with respect to the $\mathrm{NC}$ charge. The high value of the $\mathrm{NC}$ charge causes a decrease in the shadow. For low values of the charge, the shape deviates from the standard circle. Moreover, for the highly spinning BH, the shadow is more deformed than for the slowly spinning $\mathrm{BH}$. There is more deformation for $\theta_{0}=\pi / 2$ as compared to $\theta_{0}=\pi / 3$.

\subsection{Observables}

Here, we study observables for the charged rotating NC BH. The observables can be used to determine important astronomical information as regards the collapsed objects (BHs and naked singularities) such as to characterize the apparent shape of the shadow. The silhouette of the BH shadow determines apparent image of the photon capture sphere seen by the observer at large distance. Hioki and Meada [12] proposed that the shadows' silhouette passes through four points, the top $\left(\alpha_{t}, \beta_{t}\right)$, bottom $\left(\alpha_{b}, \beta_{b}\right)$, right $\left(\alpha_{r}, 0\right)$ and left point $\left(\alpha_{l}, 0\right)$. They presented two parameters as observable quantities. First of all, the radius $R_{S}$ determines the size (approximate) of the shadow defined as the radius passing through three different points, i.e., top, bottom and the most right points of a reference circle. The second is $\delta_{s}$, which measures the deformation and is defined as $\delta_{s}=\frac{D_{s}}{R_{s}}$, where $D_{s}$ is the difference between the most left points, i.e., $\left(\alpha_{l}, 0\right)$ and $\left(\tilde{\alpha}_{l}, 0\right)$. The point $\left(\tilde{\alpha}_{l}, 0\right)$ cuts the horizontal axis opposite to $\left(\alpha_{r}, 0\right)$. This discussion can be explained through the Fig. 1 given in [71]. The plots of the radius of shadow and deformation parameter are shown in Fig. 4. We observe that the deformation parameter has increasing behavior along spin for the varying values of $\mathrm{NC}$ charge. For large values of the $\mathrm{NC}$ charge, the deformation gradually decreases, which means that the shape of shadow will approximately be a circle. The deformation is high for high values of the spin. The radius of the shadow has decreasing behavior with respect to the spin.

\section{Shadow of the BH surrounded by plasma}

In this section, we explore the effects of plasma on the shadow of rotating charged $\mathrm{BH}$ in the background of $\mathrm{NC}$ geometry. For the axially symmetric $\mathrm{BH}$, the refraction index of the plasma is $n=n\left(x^{i}, w\right)$, where the photon frequency $w$ is measured by the observer with velocity $u^{v}$. The effective energy of the photon is $\hbar w=-p_{v} u^{v}$ ( $p_{v}$ is the 4-momentum of the photon). The relation between the refraction index and 4-momentum of the photon is given as [73]

$n^{2}=1+\frac{p_{\nu} p^{v}}{p_{\sigma} u^{\sigma}}$

where $n=1$ in the absence of plasma. In order to find analytical results, it is useful to write refraction index with specific plasma frequency $w_{e}$ [56] 
Fig. 3 Shadows of charged NC $\mathrm{BH}$
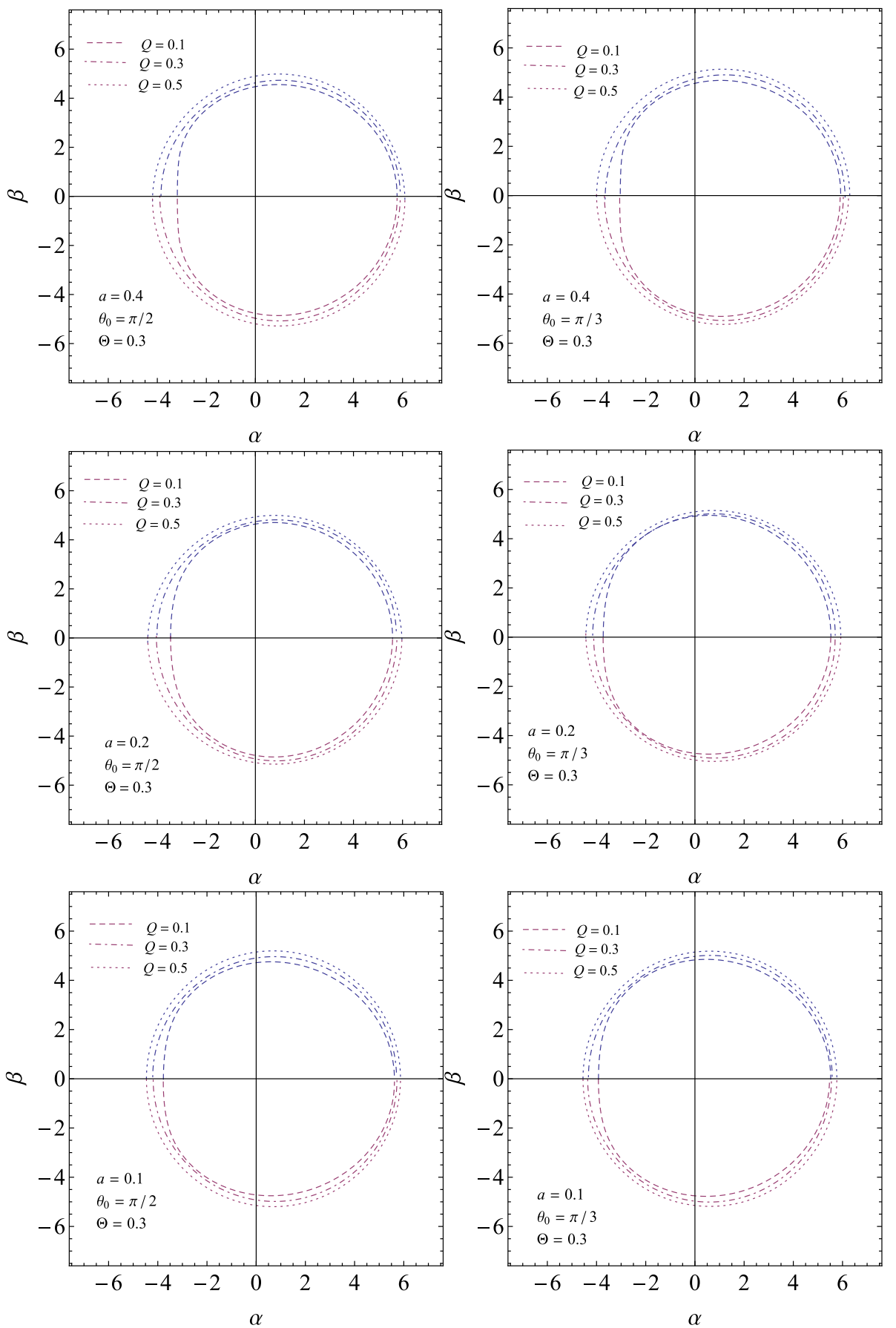

$n^{2}=1-\frac{w_{e}^{2}}{w^{2}}$

The Hamilton-Jacobi equation for the photon around the BH surrounded by a plasma has the following form $[56,73]$ :

$\frac{\partial S}{\partial \lambda}=\frac{1}{2}\left[g^{\nu \sigma} p_{\nu} p_{\sigma}-\left(n^{2}-1\right)\left(p_{0} \sqrt{-g^{00}}\right)^{2}\right]$.
This leads to the following equations of motion:

$\Sigma \frac{\partial t}{\partial \lambda}=a\left(L-a n^{2} E \sin ^{2} \theta\right)+\frac{r^{2}+a^{2}}{\Delta}\left[n^{2} E\left(r^{2}+a^{2}\right)-a L\right]$,

$\Sigma \frac{\partial \phi}{\partial \lambda}=\left(L \csc ^{2} \theta-a E\right)+\frac{a}{\Delta}\left[E\left(r^{2}+a^{2}\right)-a L\right]$,

$\Sigma \frac{\partial r}{\partial \lambda}= \pm \sqrt{R_{p}}$,

$\Sigma \frac{\partial \theta}{\partial \lambda}= \pm \sqrt{\Theta_{p}}$, 

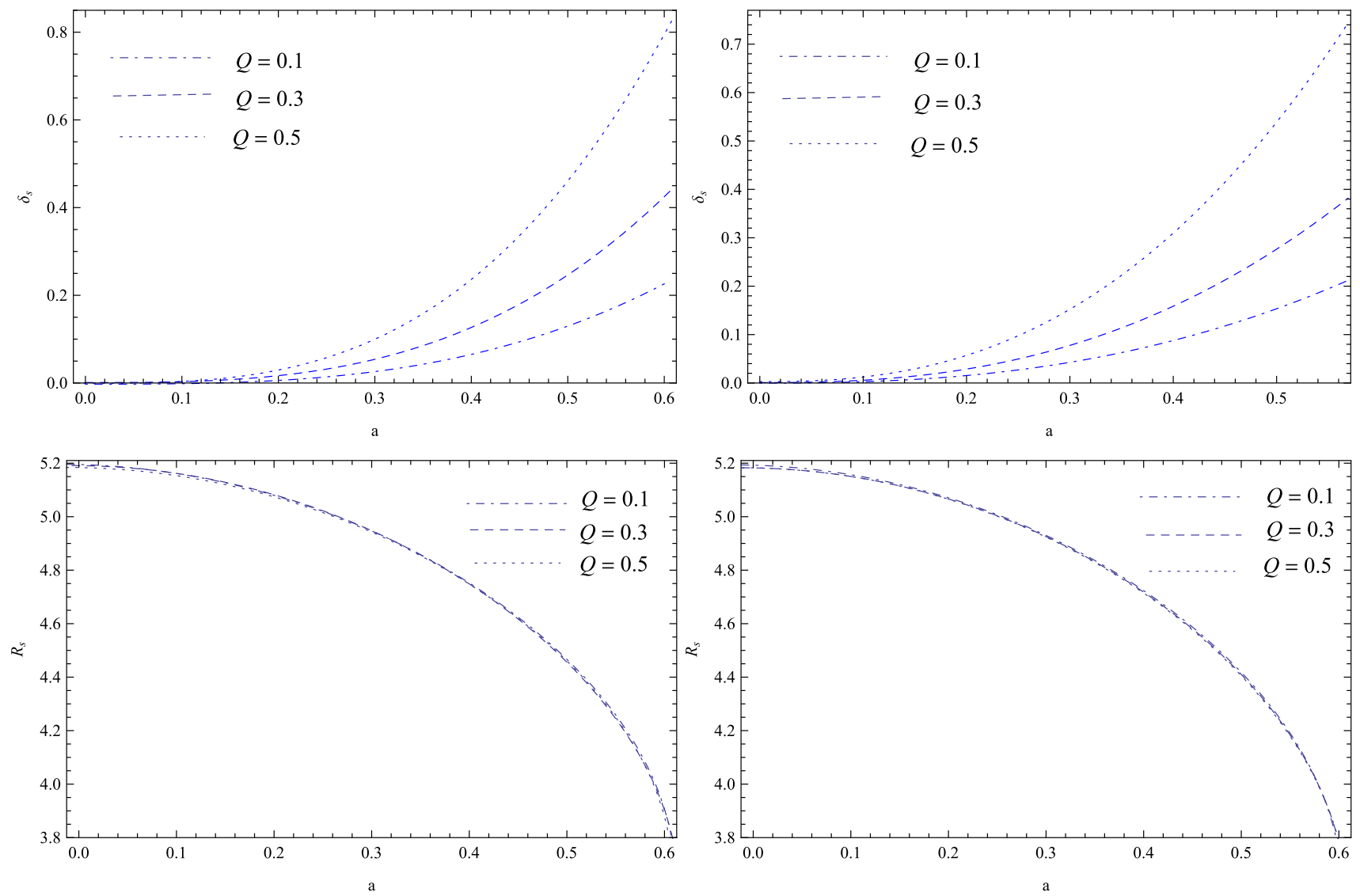

Fig. 4 The upper panel and lower panel shows deformation parameter and radius of the shadow corresponding to $\pi / 2$ (left), $\pi / 3$ (right) and $\Theta=0.3$

where

$$
\begin{aligned}
R_{p}= & {\left[E\left(r^{2}+a^{2}\right)-a L\right]^{2}+\left(n^{2}-1\right)\left(r^{2}+a^{2}\right)^{2} E^{2} } \\
& -\Delta\left[K+(L-a E)^{2}\right], \\
\Theta_{p}= & K+\cos ^{2} \theta\left(a^{2} E^{2}-L^{2} \csc ^{2} \theta\right)-a^{2}\left(n^{2}-1\right) E^{2} \sin ^{2} \theta .
\end{aligned}
$$

For the sake of simplicity, we chose a specific form of $w_{e}$ with the assumption that the spacetime is filled with cold plasma (non-magnetized) whose electron plasma frequency is a function of the radial coordinate,

$w_{e}^{2}=\frac{4 \pi e^{2} N(r)}{m_{e}}$,

where $e$ and $m_{e}$ represent the electron charge and mass, respectively, and $N(r)$ denotes the number density of the electrons in plasma. Following [56], we consider $N(r)$ as the power law density such that $N(r)=N_{0} / r^{h}, h \geq 0$, which yields $w_{e}^{2}=k / r^{h}$, where $k$ is an arbitrary constant. Here we take $h=1$. To study the effect of plasma on the shadow of a charged rotating $\mathrm{NC} \mathrm{BH}$, we follow the same procedure as in Sect. 2. We find the parameters $\xi$ and $\eta$ of the form

$$
\begin{aligned}
\xi & =\frac{Y}{X}+\sqrt{\frac{Y^{2}}{X^{2}}-\frac{Z}{X}}, \\
\eta & =\frac{\left(r^{2}+a^{2}-a \xi\right)^{2}+\left(n^{2}-1\right)\left(r^{2}+a^{2}\right)^{2}}{\Delta}-(\xi-a)^{2},
\end{aligned}
$$

where

$$
\begin{aligned}
X= & \frac{a^{2}}{\Delta}, \\
Y= & \frac{a}{\Delta\left(m(r)-r-m^{\prime}(r) r-q(r) q^{\prime}(r)\right)}\left[m(r) a^{2}-m(r) r^{2}\right. \\
& \left.+m^{\prime}(r) r^{3}+m^{\prime}(r) r a^{2}+q^{2}(r) r-q(r) q^{\prime}(r)\left(r^{2}+a^{2}\right)\right], \\
Z= & \frac{n^{2}\left(r^{2}+a^{2}\right)^{2}}{\Delta}+\frac{2 r n^{2}(r)\left(r^{2}+a^{2}\right)-n(r) n^{\prime}(r)\left(r^{2}+a^{2}\right)^{2}}{\left(m(r)-r-m^{\prime}(r) r-q(r) q^{\prime}(r)\right)} .
\end{aligned}
$$

The celestial coordinates under the influence of plasma can be written as

$\alpha=-\xi \csc \theta_{0}$,

$\beta=\frac{\sqrt{\eta+a^{2} \cos ^{2} \theta_{0}-\xi^{2} \cot ^{2} \theta_{0}-n^{2} a^{2} \sin ^{2} \theta_{0}}}{n}$.

Figure 5 indicates the effect of plasma for $a=0.4, \theta=\frac{\pi}{2}$, $M=1$, and varying charge as well as $n$. We observe that 

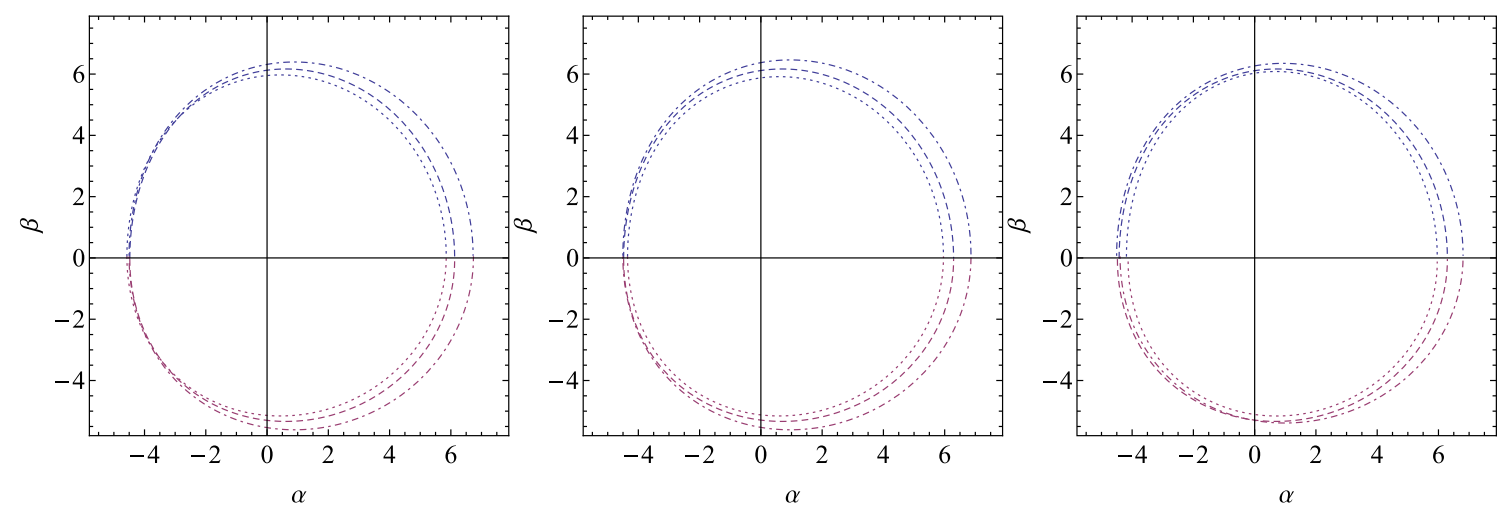

Fig. 5 Shadow of charged NC BH in the presence of plasma. Plots from left to right correspond to $Q=0.1, Q=0.3$, and $Q=0.5$ where $d o t t e d$, dashed and dotted-dashed lines correspond to the absence of plasma, $k^{2}=0.1$, and $k^{2}=0.4$

the shadow with plasma is greater than the case of without plasma. The shadow increases with the increase of plasma while an increase in the value of charge tends to decrease the deformation.

\section{Final remarks}

The study of the apparent shapes of a BH shadow has been the subject of interest for the last few years as it may be possible to observe a $\mathrm{BH}$ in the center of galaxy in near future [72]. The shadow appears due to the effects of strong gravity near the $\mathrm{BH}$, so it may be helpful to explore its nature. For classical Kerr and Kerr-Newman BHs, the shadow is affected by the spin, charge and the inclination angle. Since the NC geometry has many applications such as resolving the singularity problems in GR, it would be interesting to study the effect of NC charge parameter on the shadow as well the particle orbits. In this paper, we have examined the shadow cast by a charged rotating NC BH. In order to study the effect of NC charge on the silhouette of shadow, we have first obtained null geodesics and analyzed the behavior of the effective potential. It is found that the photons are stable for small values of the radius but they approach maxima for the large radius which correspond to unstable orbits. After reaching the maxima, $V_{\text {eff }}$ starts diverging.

We have used null geodesics to find the celestial coordinates $\alpha$ and $\beta$, which describe the nature of the shadow. It is found that the charged $\mathrm{NC} \mathrm{BH}$ shadow is not only affected by NC charge but also due to spin as well as the angle of inclination. We observe that the silhouette of shadow changes its shape with respect to varying charge parameter. The shape deviates from the circle for the small charge while for the large $\mathrm{NC}$ charge, it approximately maintains the shape of a circle. This behavior is almost similar to the rotating uncharged NC BH [39] (where the shape of the circle is maintained with increase in the NC parameter) as well as charged BHs $[74,75]$. The high values of spin as well as angle of inclination deform the shape of the shadow. We have also discussed the radius of shadow and deformation parameter for this $\mathrm{BH}$. The large value of NC charge corresponds to less deformation while for rotating uncharged $\mathrm{NC}$ $\mathrm{BH}[39]$ the distortion parameter increases with $\mathrm{NC}$ parameter. This leads to the idea that a NC charge helps to maintain the shape of a circle. The radius of the shadow decreases as spin increases. Finally, we have examined the shadow of a charged rotating $\mathrm{NC} \mathrm{BH}$ in the presence of plasma. The existence of plasma increases the size of the shadow and deformation in the shape reduces due to the presence of $\mathrm{NC}$ charge which is analogous to [75]. We conclude that the presence of an NC charge not only affects the particle orbits but also affects the shadow of the $\mathrm{BH}$.

Acknowledgements This work has been supported by the Pakistan Academy of Sciences Project. We would like to thank Alejandro Cardenas for his kind help to draw the figures.

Open Access This article is distributed under the terms of the Creative Commons Attribution 4.0 International License (http://creativecomm ons.org/licenses/by/4.0/), which permits unrestricted use, distribution, and reproduction in any medium, provided you give appropriate credit to the original author(s) and the source, provide a link to the Creative Commons license, and indicate if changes were made.

Funded by SCOAP ${ }^{3}$.

\section{References}

1. H. Falcke, F. Melia, E. Agol, Astrophys. J. 528, L13 (2000)

2. W. Cash, A. Shipley, S. Osterman, M. Joy, Nature 407, 160 (2000)

3. H. Hirabayashi et al., arXiv: astro-ph/0501020

4. S.S. Doeleman et al., Nature 455, 78 (2008)

5. S.S. Doeleman, Astrophys. J. 695, 59 (2009)

6. J.M. Bardeen, In black holes, ed. by C. Dewitt, B.S. Dewitt, (Gordon and Breach, Philadelphia, 1973)

7. J.M. Cunningham, C.T. Bardeen, Astrophys. J. 183, 237 (1973)

8. S. Chandrasekhar, The Mathematical Theory of Black Holes (Oxford University Press, Oxford, 1983)

9. P.J. Young, Phys. Rev. D 14, 3281 (1976) 
10. A. de Varies, Class. Quantum Grav. 17, 123 (2000)

11. K. Hioki, U. Miyamoto, Phys. Rev. D 78, 044007 (2008)

12. K. Hioki, K. Maeda, Phys. Rev. D 80, 024042 (2009)

13. L. Amarilla et al., Phys. Rev. D 81, 124045 (2010)

14. L. Amarilla, E.F. Eiroa, Phys. Rev. D 87, 044057 (2013)

15. A. Abdujabbarov et al., Astrophys. Space Sci. 334, 429 (2012)

16. A. Grenzebach, V. Perlick, C. Lammerzahl, Phys. Rev. D 89, 124004 (2014)

17. U. Papnoi, Phys. Rev. D 90, 024073 (2014)

18. L. Yang, Z. Li, Int. J. Mod. Phys. D 25, 1650026 (2016)

19. A. Bohn et al., Class. Quantum Grav. 32, 065002 (2015)

20. J.O. Shipley, S.R. Dolan, Class. Quantum Grav. 33, 175001 (2016)

21. T. Johannsen, D. Psaltis, Astrophys. J. 716, 187 (2010)

22. T. Johannsen, Adv. Astron. 2012, 486750 (2012)

23. T. Johannsen, Class. Quantum Grav. 33, 124001 (2016)

24. D.J. Gross, P.F. Mende, Phys. Lett. B 197, 129 (1987)

25. D.J. Gross, P.F. Mende, Nucl. Phys. B 303, 407 (1988)

26. M. Maggiore, Phys. Lett. B 304, 65 (1993)

27. P. Nicolini, Int. J. Mod. Phys. A 24, 1229 (2009)

28. S. Doplicher, K. Fredenhagen, J.E. Roberts, Phys. Lett. B 331, 39 (1994)

29. S. Doplicher, K. Fredenhagen, J.E. Roberts, Commun. Math. Phys. 172, 187 (1995)

30. M.R. Douglas, N.A. Nekrasov, Rev. Mod. Phys. 73, 977 (2001)

31. R.J. Szabo, Phys. Rept. 378, 207 (2003)

32. S. Ansoldi, P. Nicolini, A. Smailagic, E. Spallucci, Phys. Lett. B 645, 261 (2007)

33. T.G. Rizzo, J. High Energy Phys. 09, 021 (2006)

34. E. Spallucci, A. Smailagic, P. Nicolini, Phys. Lett. B 670, 449 (2009)

35. A. Smailagic, E. Spallucci, Phys. Lett. B 688, 82 (2010)

36. P. Nicolini, A. Smailagicc, E. Spalluccie, Phys. Lett. B 632, 547 (2006)

37. K. Nozari, S.H. Mehdipour, Class. Quantum Grav. 25, 175015 (2008)

38. C. Ding et al., Phys. Rev. D 83, 084005 (2011)

39. S. Wen-Wei et al., J. Cosmol. Astropart. Phys. 08, 004 (2015)

40. H. Garcia-Compean, C. Soto-Campos, Phys. Rev. D 74, 104028 (2006)

41. K. Nozari, B. Fazlpour, Mod. Phys. Lett. A 22, 2917 (2007)

42. R. Banerjee, B.R. Majhi, S. Samnta, Phys. Rev. D 77, 124035 (2008)

43. B. Zhang et al., Europhys. Lett 94, 2002 (2011)

44. Y.G. Miao, Z. Xue, S.J. Zhang, Int. J. Mod. Phys. D 21, 1250018 (2012)
45. A.E. Vazquez, E.P. Esteban, Nuovo Cim. B 119, 489 (2004)

46. R.M. Wald, Phys. Rev. D 10, 1860 (1974)

47. D.M. Chitre, C.V. Visheshwara, Phys. Rev. D 12, 1538 (1975)

48. A.R. King, J.P. Lasota, W. Kundt, Phys. Rev. D 12, 3037 (1975)

49. B. Punsly, Astrophys. J. 498, 640 (1998)

50. B. Punsly, Astrophys. J. 498, 660 (1998)

51. D.O. Muhleman, I.D. Johnston, Phys. Rev. Lett. 17, 455 (1966)

52. R.A. Breuer, J. Ehlers, Proc. R. Soc. Lond. A 370, 389 (1980)

53. R.A. Breuer, J. Ehlers, Proc. R. Soc. Lond. A 374, 65 (1981)

54. V. Perlick, Ray Optics, Fermat's Principle and Applications to General Relativity (Springer, New York, 2000)

55. V. Perlick, O.Y. Tsupko, G.S. Bisnovatyi-Kogan, Phys. Rev. D 92 , $104031(2015)$

56. A. Rogers, Mon. Not. R. Astron. Soc. 451, 4536 (2015)

57. G.S. Bisnovatyi-Kogan, O.Y. Tsupko, Gravit. Cosmol. 15, 20 (2009)

58. G.S. Bisnovatyi-Kogan, Y. Tsupko, Mon. Not. R. Astron. Soc. 404, $1790(2010)$

59. V. Morozova, B. Ahmedov, A. Tursunov, Astrophys. Space Sci. 346, 513 (2013)

60. X. Er, S. Mao, Mon. Not. R. Astron. Soc. 437, 2180 (2013)

61. Abdujabbarov, A. et al, arXiv:1512.05206v2

62. F. Atamurotov, B. Ahmedov, A. Abdujabbarov, Phys. Rev. D 92, 084005 (2015)

63. H.S. Snyder, Phys. Rev. 71, 38 (1947)

64. H.S. Snyder, Phys. Rev. 72, 68 (1947)

65. N. Seiberg, E. Witten, J. High Energy Phys. 9909, 032 (1999)

66. S. Majid, H. Ruegg, Phys. Lett. B 334, 348 (1994)

67. S. Zakrzewski, J. Phys. A 27, 2075 (1994)

68. A. Matusis, L. Susskind, N. Toumbas, J. High Eenergy Phys. 0012, $002(2000)$

69. C.S. Chu, B.R. Greene, G. Shiu, Mod. Phys. Lett. A 16, 2231 (2001)

70. L. Modesto, P. Nicolini, Phys. Rev. D 82, 104035 (2010)

71. N. Tsukamoto, Z. Li, C. Bambi, J. Cosmol. Astropart. Phys. 06, 043 (2014)

72. H. Falcke, S.B. Markoff, Class. Quantum Grav. 30, 244003 (2000)

73. J.L. Synge, Relativity: The General Theory (North-Holland, Amsterdam, 1960)

74. L. Amarilla, E.F. Eiroa, Phys. Rev. D 85, 064019 (2012)

75. A. Abdujabbarov et al., Phys. Rev. D 93, 104004 (2016)

76. B. Carter, Phys. Rev. 174, 1559 (1968) 